\title{
Effects of Nut Proportion and Storage Temperature on Some Chemical Parameters of Pistachio Nut Cream
}

\author{
Ömer Faruk Gaml1 ${ }^{1}$ and Ibrahim Hayoglu ${ }^{2}$ \\ 1. Vocational High School, Food Technology, Korkut Ata University, Bahce-Osmaniye, Turkey \\ 2. Agricultural Faculty, Food Engineering, Harran University, Sanliurfa, Turkey
}

Received: August 4, 2011 / Published: January 20, 2012.

\begin{abstract}
Pistachio nut cream is a new product that was produced by mixing powdered sugar, roasted pistachio nut, defatted powdered milk, lecithin, vanilla and margarine. Pistachio nut cream was stored at 4 and $20{ }^{\circ} \mathrm{C}$ in sealed jars. Nut creams were analyzed by chemical analysis such as peroxide values $\left(\mathrm{meq} \mathrm{kg}^{-1}\right)$, total and free fatty acidity (\%), moisture content (\%), $\mathrm{pH}, 2$-tiobarbutric acid and browning indices. Sensory evaluations of the cream were examined according to the color, odour, texture, taste, aroma and feelings by the panelists. Samples containing $10 \%$ and $15 \%$ pistachio nut that were stored at $4{ }^{\circ} \mathrm{C}$ were better and more acceptable with respect to the chemical properties of pistachio nut cream compared to the nut cream samples that were stored at $20{ }^{\circ} \mathrm{C}$.
\end{abstract}

Key words: Cream production, pistachio nut, nut proportion, quality.

\section{Introduction}

Pistachio nut is mainly grown in many regions of the world, such as Iran, USA, Turkey, Greece, and Italy. It is consumed as salted, roasted and also used in dessert production such baklava and nut cream production in Turkey especially Gaziantep and Şanliurfa region as a main ingredient. Pistachio nut contains $\mathrm{K}, \mathrm{P}$ and also various amounts of $\mathrm{Ca}, \mathrm{Mg}$, and $\mathrm{Fe}$. Pistachio nut production is 35,000 tons annually in Turkey. The different cultivars of pistachios are grown in southeast region in our country such as Siirt, Ohadi, Halebi, Kirmizi, and Uzun. Pistachio nut contains $22 \%$ of protein, $55 \%$ of fat, $18 \%$ of carbohydrate and $5 \%$ of moisture [1]. Fatty acid content of pistachio nut is $7 \%-10.5 \%$ of palmitic, $0.9 \%-2.5 \%$ of stearic, $54 \%-71 \%$ of oleic, $16 \%-35 \%$ of linoleic acid [2]. Pistachio nut is being used in the production of baklava (special type of dessert in Turkey) and nut paste. Dried pistachios are highly susceptible to lipid oxidation because of high

Corresponding author: Ömer Faruk Gaml, Ph.D., researcher, research field: nut paste production. E-mail: farukg69@gmail.com. content of lipids and lipid oxidation causes off-flavor development that concern for the shelf life stability of many confections containing pistachios [3]. Nuts are rich in chemical composition and nutrients. Beneficial effects of nuts are attributed to some characteristics of polyunsaturated/saturated fat ratio and high percentage of monounsaturated fat and high-fiber content [4]. The dessert producers prefer Kirmizi and Uzun cultivars in baklava and nut paste production, due to their special green color, flavour and texture. Pistachio nut paste is a sweet product having a semi-hard texture which is produced by mixing the ground pistachio with glucose-sucrose solution. The mixture is kneaded up to desire texture obtained [5]. Different products can be produced by using nut having different texture such as peanut paste [6], walnut paste [7], pistachio nut paste [5], hazelnut paste [8], chocolate peanut spread [9], During the production of nut containing foods, they are subjected to heat treatment and most common application is roasting of nuts. During the heat treatment especially roasting, desirable flavor and aroma development occur. Chemical and biological changes of 
nut lipids are detected due to heat treatment such as free fatty acidity, iodine, peroxide and acid values of oils [4]. Proteins of acid-soluble fraction are sensitive to the heat treatment and may have a role in flavor volatile production during the roasting process [10].

Spreadable nut cream is a new product produced by mixing powdered sugar, roasted pistachio nut, defatted milk powder, lecithin, powdered vanilla and margarine until the desire cream shape is obtained [11, 12]. The nut cream contains water, higher amount sugar and fat. Due to the components deteriorative reactions may occur during the long-term of storage of the product. The pistachio nut paste changed its color to brown at high water activities and the occurence of brown color may be due to Maillard reactions [13]. Long-terms storage of foods that contain sugar, water, fat and protein causes deteriorative reactions, browning, lipid oxidation and microbial growth [14]. The moisture effects can be explained in terms of water activity values and it is an important factor for finished food stability.

There was a similar study that was based on the pistachio nut in literature which contains glucose-sucrose mixture and pistachio nut [5]. The aim of this research was to produce a new product that contains margarine, sugar, lecithin, milk powder and vanilla by using pistachio nut having the property of spreadability within different amounts of pistachio nut and also determine the factors affecting the quality of this nut cream during the storage.

\section{Materials and Methods}

\subsection{Materials}

Pistachio nut cream was produced roasting nut and adding ingredients to the mixture. The pistachio nuts were roasted at $160-165{ }^{\circ} \mathrm{C}$ for $8-10 \mathrm{~min}$. Roasted pistachio nuts were grounded and powdered sugar and defatted powdered milk were added. Margarine, lecithin and also powdered vanilla were added to the mixture. The mixing procedure was carried out until the desire spreadable texture obtained. In the production of spreadable nut cream; $30 \%$ of sugar, $30 \%$ of margarine, $6 \%$ of defatted milk, $0.5 \%$ of lecithin and $0.5 \%$ of vanilla were used by different proportion of pistachio nut $(5 \%, 10 \%$ and $15 \%)$. The spreadable nut creams were filled in to the glass jars around $250-260 \mathrm{~g}$ within polypropylene covers and stored at 4 and $20^{\circ} \mathrm{C}$ for 240 days.

\subsection{Methods}

The pistachio nut creams were analyzed monthly for changes in chemical parameters such as peroxide values (meq $\left.\mathrm{kg}^{-1}\right)$ [15], total and free fatty acidity (\%) [16], pH [17], moisture content (\%) [18], browning indices $\left(\mathrm{A}_{420}\right)$ [19], 2-tiobarbutric acid (TBA) values $\left(\mathrm{A}_{530}\right)$ [20], total chlorophyll content [21], ash content (\%) [22], carbohydrate (\%) [8], cellulose (\%) [16] and protein content (\%) [23]. Free fatty, total acidity (\%) and $\mathrm{pH}$ are of significance with respect to taste balances of the cream and also extend of lipolysis expressed as oleic acid (\%) values.

The browning indices of the nut cream were measured absorbance value due to the experimental procedures of mixing the samples with ethyl alcohol, blended, filtered, and read the absorbance at $420 \mathrm{~nm}$.

The sensory evaluations of pistachio nut cream were done according to the parameters that affect the organoleptic properties of the cream including color, odour, texture, taste, aroma and feelings in the mouth during the storage at 4 and $20^{\circ} \mathrm{C}$. Sensory evaluation of the nut cream was carried out by the panelists.

The experimental procedures were carried out due to three replications and the averages of results were used. Analysis of variance (ANOVA) was used to determine any significant effects between the interactions at the level of 0.01 through the use of statistical analysis program (TARIST).

\section{Results and Discussions}

The pistachio nut cream contained carbohydrate, moisture, ash, protein, cellulose and fat (Table 1). The results showed that the spreadable nut cream contained 
Table 1 The composition of the spreadable pistachio nut cream.

\begin{tabular}{lccc}
\hline \multirow{2}{*}{ Ingredients (\%) } & \multicolumn{3}{c}{ Pistachio nut proportions (\%) } \\
\cline { 2 - 4 } & 5 & 10 & 15 \\
\hline Moisture & 9.66 & 9.77 & 9.81 \\
Protein & 6.22 & 7.27 & 8.22 \\
Ash & 1.20 & 1.24 & 1.25 \\
Fat & 28.17 & 29.92 & 31.02 \\
Carbohydrate & 54.08 & 50.99 & 48.69 \\
Cellulose & 0.645 & 0.792 & 0.971 \\
\hline
\end{tabular}

higher amount of carbohydrate due to sugar content. The composition of the nut cream varied due to the pistachio nut content. The changes in chemical parameters of spreadable pistachio nut cream were presented in Table 2. As depicted in Tables 3 and 4, pistachio nut proportions, storage temperatures, and storage period affected the chemical parameters of the nut cream significantly $(P<0.01)$.

The peroxide values of nut samples stored in glass jars increased due to the increasing amount of pistachio nut $(5 \%, 10 \%$ and $15 \%)$. The spreadable nut cream samples that contained $5 \%$ pistachio nut had the lowest PO values $\left(0.1695 \mathrm{meq} \mathrm{kg}^{-1}\right)$. The higher storage temperature and content of pistachio nut have resulted in higher peroxide values (Table 3). Increase in storage temperature caused an increase in total and free fatty acidity (\%) and decrease in $\mathrm{pH}$ values of pistachio nut cream.

Increase in peroxide values of the nut cream could be attributed to the oxidation of fats in the samples. Increase in storage temperatures causes the oxidation of fats containing non-saturated fatty acids such as pistachio nut paste [5] and walnut paste [7], producing alkyl peroxides during the storage periods.

The peroxide values of spreadable nut cream increased from 0.0873 to 0.5494 (meq kg ${ }^{-1}$ ) during the storage period (Fig. 1). Peroxide values were used as an indicator of the formation of hydroperoxide due to lipid oxidation for foods that contain pistachio nuts [3].

$\mathrm{pH}$ values of pistachio nut cream were different and it was reversely proportional to the pistachio nut proportion (Table 3). Increase in pistachio nut amount caused increases in total and free fatty acidity (\%) of the nut cream during the storage period. Pistachio nut proportion had affected chemical parameters significantly at a level of $P<0.01$. Storage temperature had affected significantly on the PO (meq $\left.\mathrm{kg}^{-1}\right), \mathrm{pH}$, moisture (\%) and total acidity (\%) except free fatty acidity (\%), browning indice and 2-tiobarbutric acid value (Table 3 ) at a level of $P<0.01$.

The storage temperature-storage period interaction had affected significantly on PO (meq $\mathrm{kg}^{-1}$ ), $\mathrm{pH}$, moisture content (\%), total acidity (\%) and 2-tiobarbutric acid value (Table 4) but no significant difference in browning indice and free fatty acidity (\%) of the nut cream. Maximum peroxide values of $10\left(\mathrm{meq} \mathrm{kg}^{-1}\right)$ is allowed for foods which contain fats [16]. The samples that contain $5 \%$ pistachio nut had a higher $\mathrm{pH}$ values compared to the pistachio nut cream that contain $10 \%$ and $15 \%$ pistachio nut and lower $\mathrm{pH}$ values compared to $4{ }^{\circ} \mathrm{C}$ (Table 5). $\mathrm{pH}$ values of nut samples varied during the storage period from 6.75 to 5.94 (Fig. 2).

The storage temperature-pistachio nut proportion interaction had an affect on PO (meq $\left.\mathrm{kg}^{-1}\right), \mathrm{pH}$, moisture content (\%), total and free fatty acidity (\%), browning indice and 2-tiobarbutric acid values of pistachio nut cream significantly at a level of $P<0.01$.

Moisture content of the spreadable nut cream slightly decreased and this was due to polypropylene covers which permits water vapour transmittence from the glass jars. The moisture content of the spreadable nut cream stored at $4{ }^{\circ} \mathrm{C}$ was higher than pistachio nut cream that were stored at $20^{\circ} \mathrm{C}$ (Fig. 3). The moisture content of the spreadable nut samples decreased from $9.78 \%$ to $9.38 \%$ during the storage periods. The spreadable nut cream that contains $15 \%$ pistachio nut had a higher water content than samples containing $10 \%$ and $5 \%$ pistachio nut. There was a slight decrease in water contents of the spreadable nut cream during the storage periods and this was due to polypropylene cover of the glass jars.

The spreadable nut cream that were stored at $4{ }^{\circ} \mathrm{C}$ had a lower total acidity compared with to the samples that were stored at $20^{\circ} \mathrm{C}$. Total acidity of the samples varied between $1.24 \%$ and $1.884 \%$ during the storage 
Table 2 Changes in some chemical properties of spreadable pistachio nut cream during storage at 4 and $20{ }^{\circ} \mathrm{C}$.

\begin{tabular}{|c|c|c|c|c|c|c|c|c|c|c|c|}
\hline \multirow{2}{*}{ Parameters } & \multirow{2}{*}{$\begin{array}{l}\text { Nut proportion } \\
(\%)\end{array}$} & \multirow{2}{*}{$\begin{array}{l}\text { Temperature } \\
\left({ }^{\circ} \mathrm{C}\right)\end{array}$} & \multicolumn{9}{|c|}{ Storage period (month) } \\
\hline & & & 0 & 1 & 2 & 3 & 4 & 5 & 6 & 7 & 8 \\
\hline \multirow{6}{*}{ PO (meq kg $\left.{ }^{-1}\right)$} & \multirow{2}{*}{5} & 4 & 0.077 & 0.184 & 0.266 & 0.422 & 0.433 & 0.468 & 0.469 & 0.470 & 0.495 \\
\hline & & 20 & 0.077 & 0.125 & 0.280 & 0.495 & 0.497 & 0.501 & 0.506 & 0.514 & 0.524 \\
\hline & \multirow{2}{*}{10} & 4 & 0.088 & 0.172 & 0.244 & 0.497 & 0.498 & 0.503 & 0.513 & 0.519 & 0.524 \\
\hline & & 20 & 0.088 & 0.138 & 0.294 & 0.494 & 0.494 & 0.506 & 0.515 & 0.518 & 0.534 \\
\hline & \multirow{2}{*}{15} & 4 & 0.095 & 0.124 & 0.254 & 0.488 & 0.501 & 0.514 & 0.523 & 0.543 & 0.563 \\
\hline & & 20 & 0.095 & 0.144 & 0.297 & 0.475 & 0.510 & 0.524 & 0.530 & 0.565 & 0.596 \\
\hline \multirow{6}{*}{$\mathrm{pH}$} & \multirow{2}{*}{5} & 4 & 6.72 & 6.60 & 6.23 & 6.17 & 6.12 & 6.09 & 6.08 & 6.03 & 5.99 \\
\hline & & 20 & 6.72 & 6.31 & 6.17 & 6.15 & 6.13 & 6.11 & 6.04 & 5.99 & 5.94 \\
\hline & \multirow{2}{*}{10} & 4 & 6.75 & 6.65 & 6.15 & 6.11 & 6.08 & 6.08 & 6.07 & 6.00 & 5.97 \\
\hline & & 20 & 6.75 & 6.026 & 6.06 & 5.97 & 6.00 & 5.98 & 5.96 & 5.92 & 5.89 \\
\hline & \multirow{2}{*}{15} & 4 & 6.76 & 6.67 & 6.08 & 6.10 & 6.08 & 6.06 & 6.05 & 6.00 & 5.94 \\
\hline & & 20 & 6.76 & 6.22 & 6.00 & 6.00 & 5.99 & 5.97 & 5.95 & 5.90 & 5.87 \\
\hline \multirow{6}{*}{ Moisture (\%) } & \multirow{2}{*}{5} & 4 & 9.66 & 9.62 & 9.52 & 9.48 & 9.44 & 9.44 & 9.43 & 9.40 & 9.39 \\
\hline & & 20 & 9.66 & 9.52 & 9.48 & 9.42 & 9.41 & 9.40 & 9.39 & 9.39 & 9.38 \\
\hline & \multirow{2}{*}{10} & 4 & 9.77 & 9.62 & 9.54 & 9.51 & 9.50 & 9.48 & 9.47 & 9.46 & 9.45 \\
\hline & & 20 & 9.77 & 9.68 & 9.49 & 9.41 & 9.40 & 9.40 & 9.39 & 9.38 & 9.37 \\
\hline & \multirow{2}{*}{15} & 4 & 9.81 & 9.65 & 9.57 & 9.47 & 9.46 & 9.45 & 9.45 & 9.44 & 9.43 \\
\hline & & 20 & 9.81 & 9.76 & 9.62 & 9.56 & 9.54 & 9.50 & 9.48 & 9.47 & 9.45 \\
\hline \multirow{6}{*}{$\begin{array}{l}\text { Total acidity } \\
(\%)\end{array}$} & \multirow{2}{*}{5} & 4 & 1.242 & 1.459 & 1.489 & 1.517 & 1.518 & 1.524 & 1.525 & 1.530 & 1.539 \\
\hline & & 20 & 1.242 & 1.384 & 1.444 & 1.524 & 1.528 & 1.532 & 1.541 & 1.556 & 1.573 \\
\hline & \multirow{2}{*}{10} & 4 & 1.411 & 1.402 & 1.545 & 1.696 & 1.699 & 1.703 & 1.708 & 1.710 & 1.723 \\
\hline & & 20 & 1.411 & 1.383 & 1.609 & 1.807 & 1.811 & 1.817 & 1.820 & 1.833 & 1.844 \\
\hline & \multirow{2}{*}{15} & 4 & 1.424 & 1.497 & 1.564 & 1.803 & 1.809 & 1.810 & 1.812 & 1.821 & 1.831 \\
\hline & & 20 & 1.424 & 1.497 & 1.564 & 1.803 & 1.809 & 1.810 & 1.812 & 1.821 & 1.831 \\
\hline \multirow{6}{*}{$\begin{array}{l}\text { Free fatty } \\
\text { acidity }(\%)\end{array}$} & 5 & 4 & 0.268 & 0.300 & 0.354 & 0.473 & 0.482 & 0.484 & 0.490 & 0.496 & 0.503 \\
\hline & 3 & 20 & 0.268 & 0.320 & 0.414 & 0.649 & 0.650 & 0.659 & 0.660 & 0.673 & 0.694 \\
\hline & 10 & 4 & 0.263 & 0.286 & 0.349 & 0.437 & 0.462 & 0.493 & 0.500 & 0.514 & 0.524 \\
\hline & 10 & 20 & 0.263 & 0.319 & 0.434 & 0.682 & 0.686 & 0.695 & 0.699 & 0.703 & 0.713 \\
\hline & 15 & 4 & 0.296 & 0.327 & 0.381 & 0.580 & 0.588 & 0.612 & 0.618 & 0.620 & 0.934 \\
\hline & 10 & 20 & 0.296 & 0.337 & 0.434 & 0.683 & 0.709 & 0.710 & 0.729 & 0.734 & 0.741 \\
\hline & 5 & 4 & 0.279 & 0.349 & 0.418 & 0.430 & 0.436 & 0.440 & 0.442 & 0.452 & 0.466 \\
\hline & 3 & 20 & 0.279 & 0.383 & 0.412 & 0.435 & 0.442 & 0.452 & 0.456 & 0.464 & 0.477 \\
\hline Browning & 10 & 4 & 0.359 & 0.371 & 0.402 & 0.448 & 0.458 & 0.463 & 0.467 & 0.474 & 0.484 \\
\hline indice $\left(\mathrm{A}_{420}\right)$ & 10 & 20 & 0.359 & 0.390 & 0.434 & 0.470 & 0.480 & 0.512 & 0.518 & 0.534 & 0.547 \\
\hline & 15 & 4 & 0.466 & 0.463 & 0.472 & 0.483 & 0.490 & 0.498 & 0.502 & 0.524 & 0.534 \\
\hline & 12 & 20 & 0.466 & 0.489 & 0.491 & 0.496 & 0.524 & 0.539 & 0.550 & 0.580 & 0.601 \\
\hline & 5 & 4 & 0.121 & 0.141 & 0.163 & 0.219 & 0.219 & 0.231 & 0.238 & 0.244 & 0.253 \\
\hline & $J$ & 20 & 0.121 & 0.171 & 0.224 & 0.226 & 0.227 & 0.232 & 0.242 & 0.251 & 0.261 \\
\hline 2-Tiobarbutric & 10 & 4 & 0.124 & 0.157 & 0.159 & 0.212 & 0.214 & 0.228 & 0.246 & 0.252 & 0.264 \\
\hline acid (TBA) & 10 & 20 & 0.124 & 0.204 & 0.208 & 0.223 & 0.225 & 0.235 & 0.249 & 0.268 & 0.278 \\
\hline & 15 & 4 & 0.129 & 0.141 & 0.168 & 0.228 & 0.229 & 0.243 & 0.253 & 0.261 & 0.282 \\
\hline & 13 & 20 & 0.129 & 0.222 & 0.223 & 0.223 & 0.228 & 0.249 & 0.269 & 0.291 & 0.295 \\
\hline
\end{tabular}

period (Fig. 4). The interaction of nut proportion-storage time affected $\mathrm{pH}$, moisture content (\%) and total acidity (\%) significantly at a level of $P<$
0.01 level (Table 6) and no effect on peroxide value, free fatty acidity (\%), browning indice and 2-tiobarbutric acid of pistachio nut cream. 
Table 3 The effect of storage temperature and nut proportion on the chemical properties of nut cream.

\begin{tabular}{|c|c|c|c|c|c|}
\hline \multirow{2}{*}{ Parameters } & \multicolumn{3}{|c|}{ Pistachio nut proportions (\%) } & \multicolumn{2}{|c|}{ Storage temperatures $\left({ }^{\circ} \mathrm{C}\right)$} \\
\hline & 5 & 10 & 15 & 4 & 20 \\
\hline PO (meq $\left.\mathrm{kg}^{-1}\right)$ & $0.1695^{\mathrm{c}}$ & $0.4899^{\mathrm{b}}$ & $0.5220^{\mathrm{a}}$ & $0.3867^{\mathrm{b}}$ & $0.4009^{\mathrm{a}}$ \\
\hline $\mathrm{pH}$ & $6.201^{\mathrm{a}}$ & $6.153^{\mathrm{b}}$ & $6.138^{\mathrm{c}}$ & $6.213^{\mathrm{a}}$ & $6.115^{\mathrm{b}}$ \\
\hline Moisture (\%) & $9.474^{\mathrm{c}}$ & $9.508^{\mathrm{b}}$ & $9.547^{\mathrm{a}}$ & $9.493^{\mathrm{a}}$ & $9.456^{\mathrm{b}}$ \\
\hline Total acidity (\%) & $1.472^{\mathrm{c}}$ & $1.666^{\mathrm{b}}$ & $1.736^{\mathrm{a}}$ & $1.600^{\mathrm{b}}$ & $1.640^{\mathrm{a}}$ \\
\hline Free fatty acidity $(\%)$ & $0.3287^{\mathrm{b}}$ & $0.5971^{\mathrm{a}}$ & $0.6229^{\mathrm{a}}$ & $0.5064^{\mathrm{a}}$ & $0.5260^{\mathrm{a}}$ \\
\hline Browning indice $\left(\mathrm{A}_{420}\right)$ & $0.404^{\mathrm{c}}$ & $0.479^{\mathrm{b}}$ & $0.520^{\mathrm{a}}$ & $0.468^{\mathrm{a}}$ & $0.468^{\mathrm{a}}$ \\
\hline $\operatorname{TBA}\left(\mathrm{A}_{530}\right)$ & $0.161^{\mathrm{c}}$ & $0.227^{\mathrm{b}}$ & $0.261^{\mathrm{a}}$ & $0.214^{\mathrm{a}}$ & $0.219^{\mathrm{a}}$ \\
\hline
\end{tabular}

${ }_{\mathrm{a}, \mathrm{b}, \mathrm{c}}$ Different letters indicate significant difference between the row at a level of $P<0.01$.

Table 4 The effect of storage temperature-storage period interaction on the chemical properties of nut cream.

\begin{tabular}{|c|c|c|c|c|c|c|c|c|c|c|}
\hline \multirow{2}{*}{ Parameters } & \multirow{2}{*}{$\begin{array}{l}\text { Temperature } \\
\left({ }^{\circ} \mathrm{C}\right)\end{array}$} & \multicolumn{9}{|c|}{ Storage period (month) } \\
\hline & & 0 & 1 & 2 & 3 & 4 & 5 & 6 & 7 & 8 \\
\hline \multirow[t]{2}{*}{$\mathrm{PO}\left(\right.$ meq $\left.\mathrm{kg}^{-1}\right)$} & 4 & $0.350^{b}$ & $0.350^{\mathrm{b}}$ & $0.351^{b}$ & $0.378^{\mathrm{b}}$ & $0.380^{\mathrm{b}}$ & $0.380^{b}$ & $0.427^{\mathrm{a}}$ & $0.429^{\mathrm{a}}$ & $0.430^{\mathrm{a}}$ \\
\hline & 20 & $0.363^{\mathrm{b}}$ & $0.364^{\mathrm{b}}$ & $0.364^{\mathrm{b}}$ & $0.392^{\mathrm{b}}$ & $0.394^{\mathrm{b}}$ & $0.394^{\mathrm{b}}$ & $0.442^{\mathrm{a}}$ & $0.444^{\mathrm{a}}$ & $0.445^{\mathrm{a}}$ \\
\hline \multirow[t]{2}{*}{$\mathrm{pH}$} & 4 & $6.73^{\mathrm{a}}$ & $6.64^{b}$ & $6.15^{\mathrm{c}}$ & $6.13^{d}$ & $6.10^{\mathrm{de}}$ & $6.09^{\mathrm{e}}$ & $6.06^{\mathrm{e}}$ & $6.01^{\mathrm{f}}$ & $5.97^{\mathrm{g}}$ \\
\hline & 20 & $6.75^{\mathrm{a}}$ & $6.26^{\mathrm{b}}$ & $6.07^{\mathrm{c}}$ & $6.05^{\mathrm{c}}$ & $6.04^{\text {cde }}$ & $6.02^{\mathrm{de}}$ & $5.98^{\mathrm{e}}$ & $5.94^{\mathrm{f}}$ & $5.90^{\mathrm{g}}$ \\
\hline \multirow[t]{2}{*}{ Moisture (\%) } & 4 & $9.75^{\mathrm{a}}$ & $6.93^{\mathrm{b}}$ & $9.54^{\mathrm{c}}$ & $9.49^{\mathrm{d}}$ & $9.47^{\mathrm{e}}$ & $9.46^{\mathrm{e}}$ & $9.45^{\mathrm{ef}}$ & $9.43^{\mathrm{fg}}$ & $9.42^{\mathrm{g}}$ \\
\hline & 20 & $9.70^{\mathrm{a}}$ & $9.65^{\mathrm{b}}$ & $9.53^{\mathrm{c}}$ & $9.46^{\mathrm{d}}$ & $9.45^{\mathrm{de}}$ & $9.43^{\text {ef }}$ & $9.42^{\mathrm{fg}}$ & $9.41^{\mathrm{fg}}$ & $9.40^{\mathrm{g}}$ \\
\hline \multirow{2}{*}{$\begin{array}{l}\text { Total } \\
(\%)\end{array}$} & 4 & $1.359^{\mathrm{h}}$ & $1.419^{\mathrm{g}}$ & $1.532^{\mathrm{f}}$ & $1.671^{\mathrm{e}}$ & $1.675^{\mathrm{de}}$ & $1.678^{\mathrm{cd}}$ & $1.681^{\mathrm{c}}$ & $1.688^{\mathrm{b}}$ & $1.697^{\mathrm{a}}$ \\
\hline & 20 & $1.359^{\mathrm{h}}$ & $1.450^{\mathrm{g}}$ & $1.570^{\mathrm{f}}$ & $1.728^{\mathrm{e}}$ & $1.732^{\mathrm{de}}$ & $1.736^{\mathrm{d}}$ & $1.746^{\mathrm{c}}$ & $1.756^{\mathrm{b}}$ & $1.767^{\mathrm{a}}$ \\
\hline \multirow{2}{*}{$\begin{array}{l}\text { Free fatty } \\
\text { acidity (\%) }\end{array}$} & 4 & $0.483^{\mathrm{a}}$ & $0.484^{\mathrm{a}}$ & $0.484^{\mathrm{a}}$ & $0.500^{\mathrm{a}}$ & $0.502^{\mathrm{a}}$ & $0.503^{\mathrm{a}}$ & $0.531^{\mathrm{a}}$ & $0.533^{\mathrm{a}}$ & $0.533^{\mathrm{a}}$ \\
\hline & 20 & $0.499^{\mathrm{a}}$ & $0.445^{\mathrm{a}}$ & $0.445^{\mathrm{a}}$ & $0.446^{\mathrm{a}}$ & $0.469^{\mathrm{a}}$ & $0.471^{\mathrm{a}}$ & $0.472^{\mathrm{a}}$ & $0.487^{\mathrm{a}}$ & $0.488^{\mathrm{a}}$ \\
\hline \multirow{2}{*}{$\begin{array}{l}\text { Browning indice } \\
\left(\mathrm{A}_{420}\right)\end{array}$} & 4 & $0.443^{\mathrm{a}}$ & $0.445^{\mathrm{a}}$ & $0.445^{\mathrm{a}}$ & $0.469^{\mathrm{a}}$ & $0.469^{\mathrm{a}}$ & $0.469^{\mathrm{a}}$ & $0.490^{\mathrm{a}}$ & $0.492^{\mathrm{a}}$ & $0.493^{\mathrm{a}}$ \\
\hline & 20 & $0.444^{\mathrm{a}}$ & $0.445^{\mathrm{a}}$ & $0.446^{\mathrm{a}}$ & $0.469^{\mathrm{a}}$ & $0.471^{\mathrm{a}}$ & $0.472^{\mathrm{a}}$ & $0.487^{\mathrm{a}}$ & $0.488^{\mathrm{a}}$ & $0.489^{\mathrm{a}}$ \\
\hline \multirow{2}{*}{$\begin{array}{l}\text { 2-Tiobarbutric } \\
\text { acid (TBA) }\end{array}$} & 4 & $0.195^{\mathrm{c}}$ & $0.198^{b c}$ & $0.200^{\mathrm{bc}}$ & $0.216^{\mathrm{ab}}$ & $0.218^{\mathrm{ab}}$ & $0.219^{\mathrm{ab}}$ & $0.224^{\mathrm{a}}$ & $0.226^{\mathrm{a}}$ & $0.227^{\mathrm{a}}$ \\
\hline & 20 & $0.195^{\mathrm{c}}$ & $0.200^{\mathrm{bc}}$ & $0.201^{b c}$ & $0.222^{\mathrm{ab}}$ & $0.223^{\mathrm{ab}}$ & $0.225^{\mathrm{a}}$ & $0.232^{\mathrm{a}}$ & $0.234^{\mathrm{a}}$ & $0.237^{\mathrm{a}}$ \\
\hline
\end{tabular}

$\mathrm{a}, \mathrm{b}, \mathrm{c}, \mathrm{d}, \mathrm{e}, \mathrm{f}, \mathrm{g}, \mathrm{h}$ Different letters indicate significant difference between the row at a level of $P<0.01$.

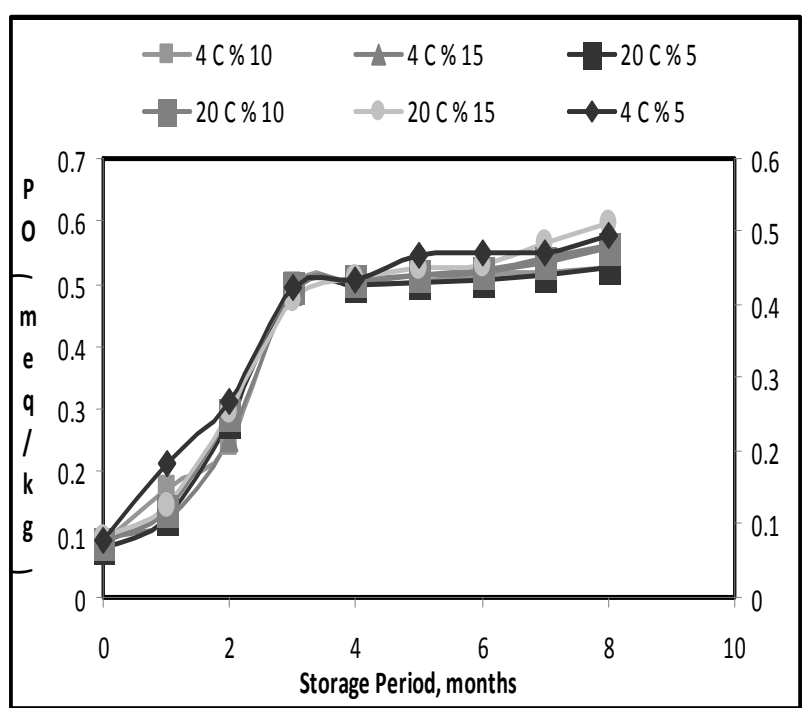

Fig. 1 Peroxide values of spreadable nut cream during storage period.
Table 5 The effect of storage temperature-nut proportion interaction on the chemical properties of nut cream.

\begin{tabular}{lccll}
\hline \multirow{2}{*}{ Parameters } & \multicolumn{2}{c}{ Temperature } & \multicolumn{3}{c}{ Pistachio nut proprtion (\%) } \\
\cline { 3 - 5 } & $\left({ }^{\circ} \mathrm{C}\right)$ & 5 & 10 & 15 \\
\hline PO $\left(\mathrm{meq}^{\mathrm{C}} \mathrm{kg}^{-1}\right)$ & 4 & $0.169^{\mathrm{c}}$ & $0.479^{\mathrm{b}}$ & $0.511^{\mathrm{a}}$ \\
& 20 & $0.169^{\mathrm{c}}$ & $0.500^{\mathrm{b}}$ & $0.532^{\mathrm{a}}$ \\
$\mathrm{pH}$ & 4 & $6.22^{\mathrm{a}}$ & $6.21^{\mathrm{a}}$ & $6.19^{\mathrm{b}}$ \\
& 20 & $6.17^{\mathrm{a}}$ & $6.09^{\mathrm{b}}$ & $6.07^{\mathrm{b}}$ \\
Moisture (\%) & 4 & $9.49^{\mathrm{b}}$ & $9.53^{\mathrm{a}}$ & $9.53^{\mathrm{a}}$ \\
& 20 & $9.45^{\mathrm{c}}$ & $9.48^{\mathrm{b}}$ & $9.56^{\mathrm{a}}$ \\
Total acidity (\%) & 4 & $1.465^{\mathrm{c}}$ & $1.628^{\mathrm{b}}$ & $1.707^{\mathrm{a}}$ \\
& 20 & $1.480^{\mathrm{c}}$ & $1.704^{\mathrm{b}}$ & $1.765^{\mathrm{a}}$ \\
Free fatty acidity & 4 & $0.327^{\mathrm{b}}$ & $0.585^{\mathrm{a}}$ & $0.606^{\mathrm{a}}$ \\
(\%) & 20 & $0.330^{\mathrm{b}}$ & $0.608^{\mathrm{a}}$ & $0.639^{\mathrm{a}}$ \\
Browning indice & 4 & $0.403^{\mathrm{c}}$ & $0.479^{\mathrm{b}}$ & $0.522^{\mathrm{a}}$ \\
& 20 & $0.406^{\mathrm{c}}$ & $0.480^{\mathrm{b}}$ & $0.518^{\mathrm{a}}$ \\
2-Tiobarbutric & 4 & $0.155^{\mathrm{c}}$ & $0.228^{\mathrm{b}}$ & $0.258^{\mathrm{a}}$ \\
acid & 20 & $0.167^{\mathrm{c}}$ & $0.226^{\mathrm{b}}$ & $0.263^{\mathrm{a}}$ \\
\hline
\end{tabular}

a, b, c Different letters indicate significantly difference between the row at a level of $P<0.01$. 


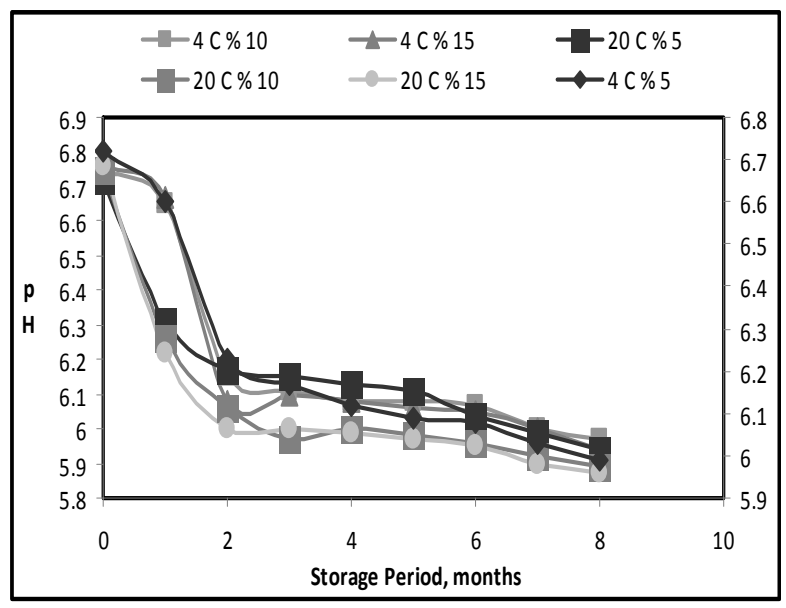

Fig. $2 \mathrm{pH}$ values of spreadable nut cream during the during the storage period.

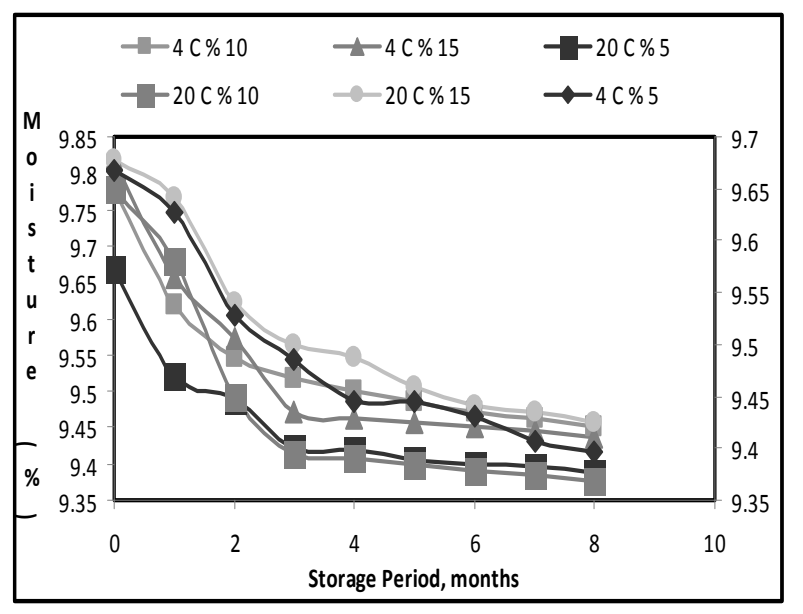

Fig. 3 Moisture content (\%) of spreadable nut cream during the storage period.

Table 6 The effect of nut proportion-storage period interaction on the chemical properties of nut cream.

\begin{tabular}{|c|c|c|c|c|c|c|c|c|c|c|}
\hline \multirow{2}{*}{ Parameters } & \multirow{2}{*}{$\begin{array}{l}\text { Nut proportion } \\
(\%)\end{array}$} & \multicolumn{9}{|c|}{ Storage period (month) } \\
\hline & & 0 & 1 & 2 & 3 & 4 & 5 & 6 & 7 & 8 \\
\hline \multirow[t]{3}{*}{$\mathrm{PO}\left(\right.$ meq $\left.\mathrm{kg}^{-1}\right)$} & 5 & $0.086^{\mathrm{c}}$ & $0.087^{\mathrm{c}}$ & $0.087^{\mathrm{c}}$ & $0.147^{b}$ & $0.147^{b}$ & $0.149^{b}$ & $0.271^{\mathrm{a}}$ & $0.273^{\mathrm{a}}$ & $0.274^{\mathrm{a}}$ \\
\hline & 10 & $0.477^{\mathrm{a}}$ & $0.478^{\mathrm{a}}$ & $0.480^{\mathrm{a}}$ & $0.489^{\mathrm{a}}$ & $0.491^{\mathrm{a}}$ & $0.491^{\mathrm{a}}$ & $0.498^{\mathrm{a}}$ & $0.500^{\mathrm{a}}$ & $0.501^{\mathrm{a}}$ \\
\hline & 15 & $0.505^{\mathrm{a}}$ & $0.507^{\mathrm{a}}$ & $0.507^{\mathrm{a}}$ & $0.519^{\mathrm{a}}$ & $0.521^{\mathrm{a}}$ & $0.523^{\mathrm{a}}$ & $0.535^{\mathrm{a}}$ & $0.538^{\mathrm{a}}$ & $0.538^{\mathrm{a}}$ \\
\hline \multirow[t]{3}{*}{$\mathrm{pH}$} & 5 & $6.71^{\mathrm{a}}$ & $6.45^{\mathrm{b}}$ & $6.20^{\mathrm{c}}$ & $6.16^{\mathrm{d}}$ & $6.13^{\mathrm{de}}$ & $6.10^{\mathrm{e}}$ & $6.06^{\mathrm{f}}$ & $6.01^{\mathrm{g}}$ & $5.97^{\mathrm{h}}$ \\
\hline & 10 & $6.75^{\mathrm{a}}$ & $6.45^{\mathrm{b}}$ & $6.08^{\mathrm{c}}$ & $6.06^{\mathrm{cd}}$ & $6.06^{\mathrm{cd}}$ & $6.04^{\mathrm{de}}$ & $6.01^{\mathrm{f}}$ & $5.96^{\mathrm{g}}$ & $5.93^{\mathrm{h}}$ \\
\hline & 15 & $6.77^{\mathrm{a}}$ & $6.44^{\mathrm{b}}$ & $6.05^{\mathrm{c}}$ & $6.04^{\mathrm{cd}}$ & $6.04^{\mathrm{cd}}$ & $6.02^{\mathrm{d}}$ & $6.00^{\mathrm{e}}$ & $5.95^{\mathrm{f}}$ & $5.91^{\mathrm{g}}$ \\
\hline \multirow[t]{3}{*}{ Moisture (\%) } & 5 & $9.66^{\mathrm{a}}$ & $9.57^{\mathrm{b}}$ & $9.50^{\mathrm{c}}$ & $9.45^{\mathrm{d}}$ & $9.43^{\mathrm{de}}$ & $9.42^{\mathrm{e}}$ & $9.41^{\mathrm{ef}}$ & $9.40^{\mathrm{f}}$ & $9.39^{\mathrm{f}}$ \\
\hline & 10 & $9.77^{\mathrm{a}}$ & $9.65^{\mathrm{b}}$ & $9.51^{\mathrm{c}}$ & $9.46^{\mathrm{d}}$ & $9.45^{\mathrm{de}}$ & $9.44^{\mathrm{de}}$ & $9.43^{\mathrm{ef}}$ & $9.42^{\mathrm{f}}$ & $9.41^{\mathrm{f}}$ \\
\hline & 15 & $9.74^{\mathrm{a}}$ & $9.71^{\mathrm{b}}$ & $9.59^{c}$ & $9.51^{\mathrm{d}}$ & $9.50^{\mathrm{de}}$ & $9.48^{\mathrm{de}}$ & $9.46^{\mathrm{ef}}$ & $9.45^{\mathrm{f}}$ & $9.44^{\mathrm{g}}$ \\
\hline \multirow{3}{*}{$\begin{array}{l}\text { Total acidity } \\
(\%)\end{array}$} & 5 & $1.242^{\mathrm{h}}$ & $1.342^{\mathrm{g}}$ & $1.466^{\mathrm{f}}$ & $1.520^{\mathrm{e}}$ & $1.522^{\mathrm{de}}$ & $1.527^{\mathrm{cd}}$ & $1.533^{\mathrm{c}}$ & $1.543^{\mathrm{b}}$ & $1.556^{\mathrm{a}}$ \\
\hline & 10 & $1.411^{\mathrm{h}}$ & $1.421^{\mathrm{g}}$ & $1.576^{\mathrm{f}}$ & $1.751^{\mathrm{e}}$ & $1.754^{\mathrm{de}}$ & $1.760^{\mathrm{cd}}$ & $1.764^{\mathrm{c}}$ & $1.773^{\mathrm{b}}$ & $1.783^{\mathrm{a}}$ \\
\hline & 15 & $1.424^{\mathrm{f}}$ & $1.540^{\mathrm{e}}$ & $1.611^{\mathrm{d}}$ & $1.829^{\mathrm{c}}$ & $1.833^{c}$ & $1.835^{\mathrm{c}}$ & $1.845^{\mathrm{b}}$ & $1.850^{\mathrm{b}}$ & $1.857^{\mathrm{a}}$ \\
\hline \multirow{3}{*}{$\begin{array}{l}\text { Free fatty } \\
\text { acidity }(\%)\end{array}$} & 5 & $0.275^{\mathrm{b}}$ & $0.276^{\mathrm{b}}$ & $0.276^{\mathrm{b}}$ & $0.312^{\mathrm{ab}}$ & $0.316^{\mathrm{ab}}$ & $0.317^{\mathrm{ab}}$ & $0.392^{\mathrm{a}}$ & $0.396^{\mathrm{a}}$ & $0.396^{\mathrm{a}}$ \\
\hline & 10 & $0.587^{\mathrm{a}}$ & $0.588^{\mathrm{a}}$ & $0.589^{\mathrm{a}}$ & $0.595^{\mathrm{a}}$ & $0.596^{\mathrm{a}}$ & $0.597^{\mathrm{a}}$ & $0.606^{\mathrm{a}}$ & $0.606^{\mathrm{a}}$ & $0.606^{\mathrm{a}}$ \\
\hline & 15 & $0.611^{\mathrm{a}}$ & $0.612^{\mathrm{a}}$ & $0.612^{\mathrm{a}}$ & $0.621^{\mathrm{a}}$ & $0.621^{\mathrm{a}}$ & $0.622^{\mathrm{a}}$ & $0.634^{\mathrm{a}}$ & $0.635^{\mathrm{a}}$ & $0.636^{\mathrm{a}}$ \\
\hline \multirow{3}{*}{$\begin{array}{l}\text { Browning } \\
\text { indice }\left(\mathrm{A}_{420}\right)\end{array}$} & 5 & $0.367^{\mathrm{a}}$ & $0.369^{\mathrm{a}}$ & $0.369^{\mathrm{a}}$ & $0.407^{\mathrm{a}}$ & $0.408^{\mathrm{a}}$ & $0.408^{\mathrm{a}}$ & $0.436^{\mathrm{a}}$ & $0.496^{\mathrm{a}}$ & $0.498^{\mathrm{a}}$ \\
\hline & 10 & $0.459^{\mathrm{a}}$ & $0.459^{\mathrm{a}}$ & $0.463^{\mathrm{a}}$ & $0.481^{\mathrm{a}}$ & $0.481^{\mathrm{a}}$ & $0.483^{\mathrm{a}}$ & $0.494^{\mathrm{a}}$ & $0.496^{\mathrm{a}}$ & $0.498^{\mathrm{a}}$ \\
\hline & 15 & $0.504^{\mathrm{a}}$ & $0.505^{\mathrm{a}}$ & $0.506^{\mathrm{a}}$ & $0.518^{\mathrm{a}}$ & $0.521^{\mathrm{a}}$ & $0.522^{\mathrm{a}}$ & $0.535^{\mathrm{a}}$ & $0.535^{\mathrm{a}}$ & $0.536^{\mathrm{a}}$ \\
\hline \multirow{3}{*}{$\begin{array}{l}\text { 2-Tiobarbutric } \\
\text { acid (TBA) }\end{array}$} & 5 & $0.121^{b}$ & $0.125^{\mathrm{b}}$ & $0.128^{\mathrm{b}}$ & $0.175^{\mathrm{a}}$ & $0.177^{\mathrm{a}}$ & $0.179^{\mathrm{a}}$ & $0.181^{\mathrm{a}}$ & $0.182^{\mathrm{a}}$ & $0.183^{\mathrm{a}}$ \\
\hline & 10 & $0.219^{\mathrm{a}}$ & $0.221^{\mathrm{a}}$ & $0.221^{\mathrm{a}}$ & $0.224^{\mathrm{a}}$ & $0.224^{\mathrm{a}}$ & $0.224^{\mathrm{a}}$ & $0.232^{\mathrm{a}}$ & $0.236^{\mathrm{a}}$ & $0.241^{\mathrm{a}}$ \\
\hline & 15 & $0.246^{\mathrm{a}}$ & $0.249^{\mathrm{a}}$ & $0.253^{\mathrm{a}}$ & $0.259^{\mathrm{a}}$ & $0.261^{\mathrm{a}}$ & $0.263^{\mathrm{a}}$ & $0.269^{\mathrm{a}}$ & $0.273^{\mathrm{a}}$ & $0.275^{\mathrm{a}}$ \\
\hline
\end{tabular}

$\mathrm{a}, \mathrm{b}, \mathrm{c}, \mathrm{d}, \mathrm{e}, \mathrm{f}, \mathrm{g}, \mathrm{h}$ Different letters indicate significantly difference between the row at a level of $P<0.01$.

The nut cream containing 5\% pistachio had lower free fatty acidity compared to samples that contained $10 \%$ and $15 \%$ pistachio (Table 2 ). Free fatty acidity of the spreadable nut cream varied between $0.2758 \%$ and $0.6553 \%$ as an oleic acid content during the storage period (Fig. 5). There was a sharp increase in free acidity until the third period; afterwards there was slight increase in free acidity of the nut samples. Similarly, the free fatty acidity (\%) and total acidity (\%) of pistachio nut (dessert type) [5] and walnut paste samples [7] increased during the storage period. Increase in storage temperature caused an increase in total and free fatty acidity (\%) of the samples. 


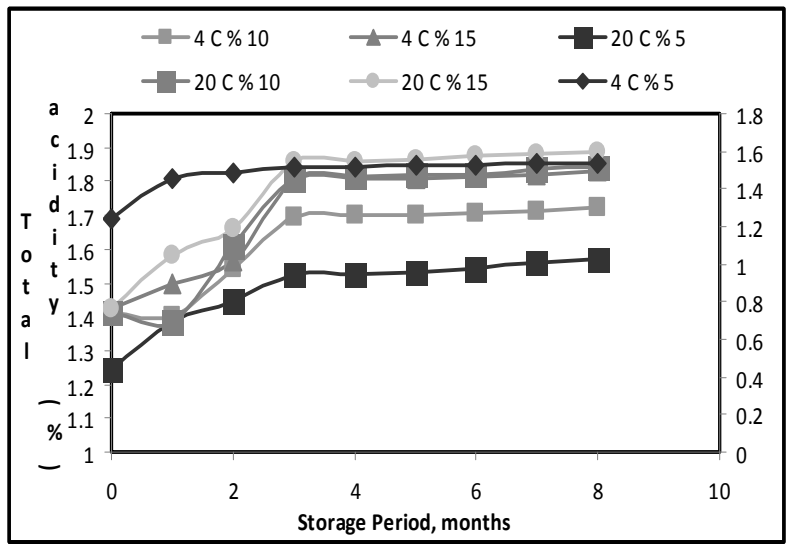

Fig. 4 Total acidity (\%) values of spreadable nut cream during the storage period.

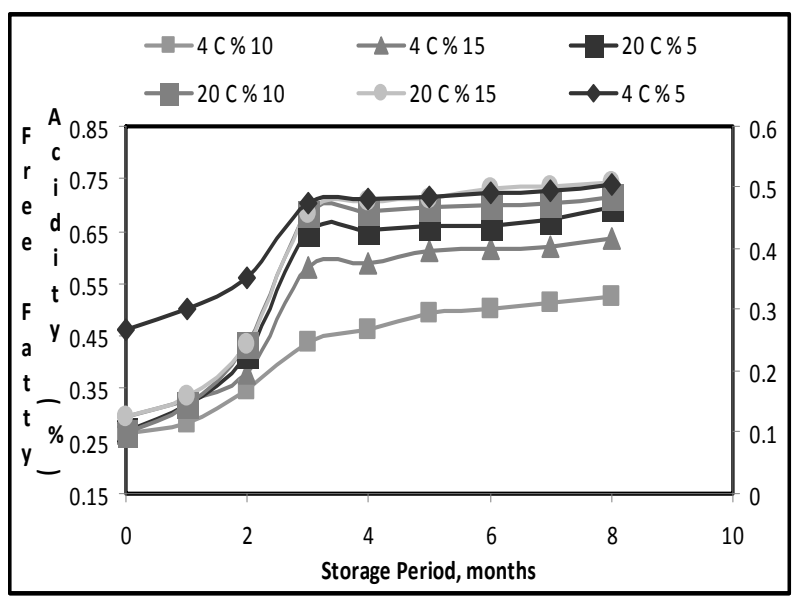

Fig. 5 Free fatty acidity (\%) values of spreadable nut cream during the storage period $\left(\mathrm{A}_{420}\right)$.

The browning indices of spreadable nut cream $\left(\mathrm{A}_{420}\right)$ varied from 0.369 to 0.532 during the storage period. The nut cream that contain 5\% pistachio nut had a lower browning indices compared to samples that contain $10 \%$ and 15\% pistachio nut (Fig. 6). Foods that contain higher amount of sugar and protein, browning reactions may occur due to Maillard reactions in pistachio nut cream at higher water activity values and also higher storage temperatures with time [13]. It is reported that there is a relation between an increase in total acidity and temperature meanwhile the changes in color from green to yellow- dirty green (formation of pheophytin) [24].

2-Tiobarbutric acid (TBA) values of the spreadable nut cream $\left(\mathrm{A}_{530}\right)$ varied from 0.121 to 0.275 during the storage period at 4 and $20^{\circ} \mathrm{C}$ (Fig. 7). TBA is used as reagent to indicate rancidity in the food materials that

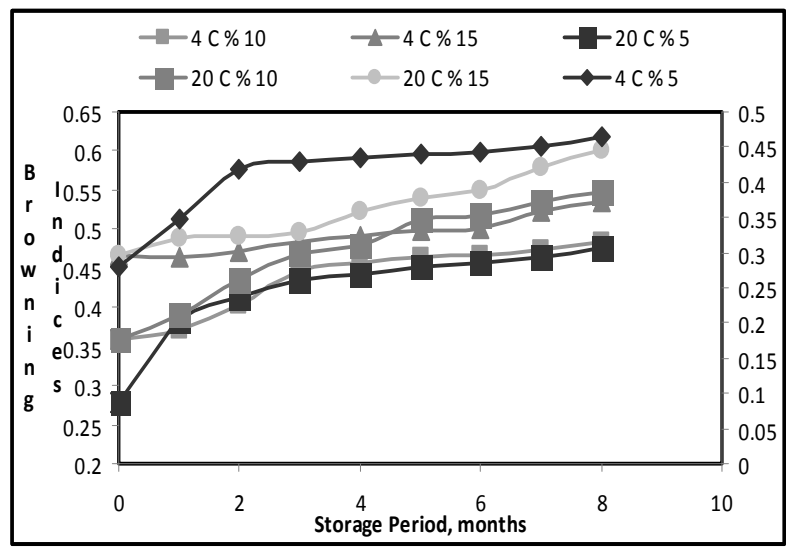

Fig. 6 Browning indices of spreadable nut cream during the storage period.

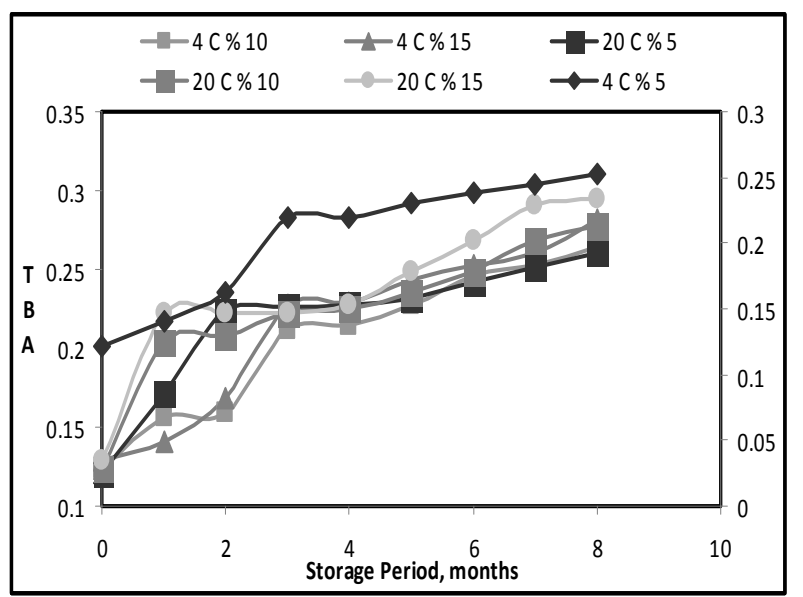

Fig. 7 2-Tiobarbutric acid values of nut cream during the storage period.

contain polyunsaturated fatty acids. It condenses with malonaldehydes that a product of linoleate producing a pink color and reacting with aldehydes causing orange color [20]. TBA test is commonly used for the edibility of the fatty foods such as walnut and pistachio nut paste. Pistachio nut cream that contain $5 \%$ proportion of nut had lower TBA values. Increase in pistachio content caused an increase in TBA values of pistachio nut cream. The relationship was statistically significant $(P$ $<0.01)$ in pistachio amounts in general. TBA values of the spreadable nut sample were close to each other during the storage period.

Spreadable pistachio nut cream is nutritious and preferred product among the breakfast foods especially children. A new product was produced at different pistachio amounts and some chemical parameters were 
Table 7 Averages of sensory evaluations of different proportion of nut cream during the storage period.

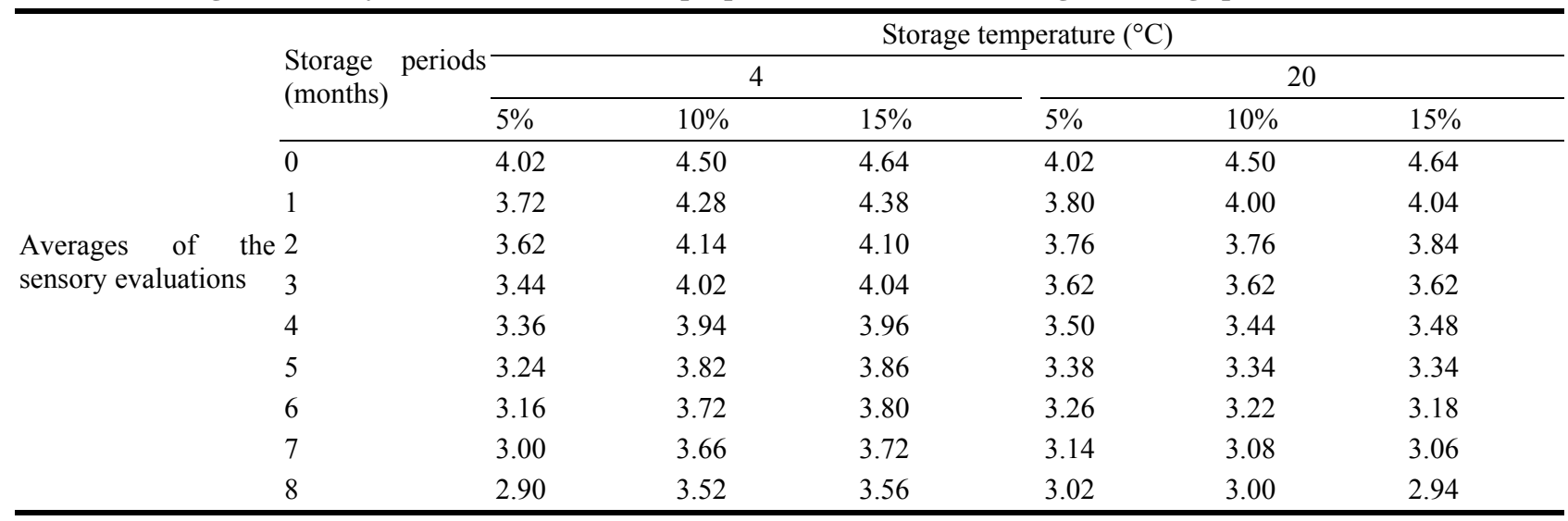

examined during the storage period of the nut samples. Spreadable nut samples that contain $10 \%$ and $15 \%$ pistachio nut gave better results in chemical parameters, so it can be produced at this proportions (10\% and $15 \%$ pistachio nut). Nut samples at $4{ }^{\circ} \mathrm{C}$ were more acceptable compared to that were stored at $20{ }^{\circ} \mathrm{C}$ due to low deteriorative reactions. Sensory evaluations of the spreadable nut cream were examined monthly according to the color, odour, texture, taste-aroma and feelings in the mouth by panelists. The averages of the sensory evaluations of the nut cream were varied due to different pistachio amounts in the spreadable nut cream (Table 7).

Especially storage periods and temperatures, the averages in the sensory evaluations decreased from the fourth period. Changes in color were considered as a negative effect by the panelists and for this reason the averages of the spreadable nut cream decreased.

\section{Conclusion}

According to the results of some chemical parameters, spreadable nut cream that were stored at $4{ }^{\circ} \mathrm{C}$ had lower peroxide values (meq $\mathrm{kg}^{-1}$ ) and total acidity $(\%)$ compared to the nut cream that stored at $20{ }^{\circ} \mathrm{C}$. Storage temperature, pistachio proportion and storage period affected chemical parameters of nut cream significantly $(P<0.01)$. Samples containing $10 \%$ and $15 \%$ pistachio nut were more acceptable due to sensory evaluations. The creams that contain $10 \%$ and $15 \%$ of pistachio nut are advisable as a new product and it is tought for supplying to the economical incomes to the producers.

\section{References}

[1] M. Pala, M. Yıldız, F. Açkurt, M. Löker, The composition of pistachio nut that grown in Turkey, Journal of Food Technology 6 (19) (1994) 405-409.

[2] E. Küçüköner, B. Yurt, Chemical composition and fatty acid contents of pistachio nuts produced in Turkey, GAP II Sgricultural Congress Book, Şanliurfa (2001) 345-352.

[3] M. Javanmard, Shelf life of whey protein-coated pistachio kernel, Journal of Food Process Engineering 31 (2008) 247-259.

[4] N. Çağlaırmak, A.C. Batkan, Nutrients and biochemistry of nuts in different consumption types in Turkey, J. Food Proc. Preserv 29 (2005) 407-423.

[5] O.F. Gamlı, I. Hayoğlu, The effect of the different packaging and storage conditions on the quality of pistachio nut paste, Journal of Food Engineering 78 (2007) 443-448.

[6] L.R. Beuchat, Relationship of water activity to moisture content in tree nuts, Journal of Food Quality 29 (6) (2006) 643-657.

[7] B. Torun, The Production Methods of Walnut Cream, Improving the Shelf Life and Quality, Institute of Natural and Applied Science, Mediterrian Univ. Antalya, 1999.

[8] S.N. Ercan, M. Dervisoğlu, Study of the steady flow behavior of hazelnut paste, Journal of Food Process Engineering 21 (1) (1998) 81-190.

[9] C.A. Chu, A.V.A. Resureccion, Sensory profiling and characterization of chocolate peanut spread using response surface methodology, Journal of Sensory Studies 20 (2005) 243-274. 


\section{Effects of Nut Proportion and Storage Temperature on Some Chemical Parameters of Pistachio Nut Cream}

[10] A.P. Adebiyi, I.A. Adeyemi, A.O. Olurundu, Effects of processing conditions and packaging material on quality attributes of dry-roasted peanuts, Journal of Food Science Agric. 13 (2000) 1465-1471.

[11] A. Altan, Special Products Production, Agricultural Faculty, Çukurova University, Adana, Turkey, 1992.

[12] O. Y1lmaz, Production of Chocolate, The General Research and Progressive Headmaster, The Industry and Trade Ministry, Ankara, Turkey, 1997.

[13] M. Maskan, F. Göğüş, The fittings of various models to water sorption isotherms of pistachio nut paste, Journal of Food Engineering 33 (1997) 227-237.

[14] J.M. Jay, Modern Food Microbiology, 3rd ed., Van Nostrand Reinhold, New York, 1986, pp. 40-41.

[15] AOAC, Official Methods of Analysis, Association of Official Analytical Chemists International, Arlington, VA, 1999.

[16] S. Nas, H.Y. Gökalp, M. Ünsal, Vegetable Oil Technology, The Pub. of Faculty of Agriciulture, Atatürk Univ. Erzurum, No.723, 1992.

[17] H. Özkaya, B. Kahveci, The Methods of Analysis of
Cereal Products, Food Technology Pub. Ankara, 1990.

[18] AOAC, Official Methods of Analysis, Association of Official Agricultural Chemists, 11th ed., 1990, pp. 1015-1017.

[19] M. Shaul, S. Israel, J.K. Isaisa, Browning determination in citrus product, Journal of Agricultural and Food Chemistry 25 (3) (1977) 179-186.

[20] C.J. Rudolph, G.V. Odell, Chemical changes in pecan oils during oxidation, Journal of Food Quality 15 (1992) 279-293.

[21] W.A. Gould, Food Quality Assurance, The Avi. Publishing Company, Wesport, Connecticut (1976) 356-361.

[22] T. Altuğ, G. Ova, K. Demirağ, U. Kurtcan, Food Quality Control, 157, Aegean Univ. Engineering Pub. No. 29 İzmir, 1995.

[23] A. Elgün, M. Certel, Z. Ertugay, H.G. Kotanc1lar, The Analytical Quality Controls and Laboratory Application Manuals for Agricultural Products, Faculty of Agriculture, Atatürk Univ., Erzurum, 1998, pp. 56-58

[24] B. Cemeroğlu, J. Acar, Fruit and Vegetable Processing Thecnology, Ankara, 1986. 